\title{
Neonatal Sepsis 1991-2001: Prevalent Bacterial Agents and Antimicrobial Susceptibilities in Bahrain
}

\author{
Khalid M. Bindayna ${ }^{a}$ Afaf Jamsheer $^{\mathrm{b}}$ Eman Farid $^{\mathrm{b}}$ Giuseppe A. Botta $^{\mathrm{a}-\mathrm{c}}$ \\ ${ }^{a}$ Department of Microbiology, Immunology and Infectious Diseases, College of Medicine and Medical Sciences, \\ Arabian Gulf University, and ${ }^{\text {b}}$ Salmaniya Medical Complex, Manama, Kingdom of Bahrain; \\ ${ }^{\mathrm{c}}$ Department of Medical Research, University of Udine, Udine, Italy
}

\section{Key Words}

Neonatal sepsis, Bahrain · Group B streptococci •

Yeasts - Antibiotic resistance

\begin{abstract}
Objectives: To investigate the organisms causing neonatal sepsis and their modifications over an extended period, to assess their changing sensitivities to antibiotics and to verify whether the policy for screening pregnant women for group B streptococci (GBS) carriage is desirable in our settings. Subjects and Methods: Medical records of all infants with positive blood culture from the Neonatal Intensive Care Unit at Salmaniya Medical Complex between 1991 and 2001 and Bahrain Defense Force Hospital between 1999 and 2001 were reviewed. Results: Of the 7,978 neonates in both hospitals 335 (4.19\%) had culture-proven bacteremia. Gram-positive bacteria were isolated at constant rate over the 11-year period. The main agents isolated were coagulase-negative Staphylococcus (CoNS) in 138 cases (41\%), Staphylococcus aureus in 28 newborns (8\%) and GBS in 26 patients $(7.8 \%$, $0.2 / 1,000$ live births). All of them were sensitive to penicillin $\mathrm{G}$, erythromycin and clindamycin. Gram-negative bacteria were declining but Escherichia coli was isolated in 35 cases $(10 \%)$. Of special concern is the increasing percentage $(5.7 \%)$ of Candida isolation. No clear trend
\end{abstract}

\section{KARGER}

Fax +4161306 1234

E-Mail karger@karger.ch

www.karger.com
(C) 2006 S. Karger AG, Basel

$1011-7571 / 06 / 0152-0131 \$ 23.50 / 0$

Accessible online at:

www.karger.com/mpp toward increasing resistance was observed, although a major difference among the two institutions was evident. Klebsiella and Enterobacter spp. showed resistance to many of the antibiotics tested, thereby posing difficult therapeutic choices. Conclusion: Good quality specimens are essential to evaluate the role of CoNS. The increasing threat of fungal infection must be carefully tackled. Specifically tailored policies for GBS prevention must be defined according to the local epidemiology.

Copyright (c) 2006 S. Karger AG, Basel

\section{Introduction}

Neonatal sepsis continues to be an important cause of morbidity and mortality both in term and in low-birthweight infants despite advances in healthcare [1-3]. Neonatal infection is of either early or late onset, defined by development of symptoms before or after the 1st week of life. Group B streptococci (GBS, Streptococcus agalacti$a e$ ) infection remains a common cause, although significantly declining [3], of early onset of neonatal infection at least in the USA [2-4], and is an increasing problem in developing countries [5]. However, in both the Arabian Gulf and the Indian subcontinent region the etiological picture of neonatal sepsis seems to differ substantially 
[6-8]. In Saudi Arabia [6, 7] Staphylococci were the predominant organisms with the majority being Staphylococcus epidermidis. In India, Staphylococcus aureus and gram-negative bacteria were the most predominant pathogens causing neonatal sepsis [8]. Several studies have documented an increase in neonatal sepsis due to gram-negative organisms [9-11]. There are no data on neonatal sepsis in the Kingdom of Bahrain.

The main aim of this study is to determine the prevalent bacterial agents of neonatal sepsis and its modification over the study period, to assess their changing sensitivities to antibiotics. Furthermore, our goal was to assess the role played by GBS in this population in order to appraise the need of introducing the universal prenatal screening to detect vaginal and rectal GBS colonization as recently recommended by Centers of Disease Control (CDC) [4].

\section{Subjects and Methods}

This retrospective study covered a period of 11 years (January 1991-December 2001) for Salmaniya Medical Complex (SMC) and 3 years for Bahrain Defense Force Hospital (BDF). The SMC has 1,000 beds while BDF has 420 beds; both constitute the two major hospitals in the Kingdom of Bahrain. The vast majority of the positive blood cultures were from Bahraini children. The blood culture registers of the Microbiology Laboratory in each hospital were reviewed to identify the positive blood cultures from the Neonatal Intensive Care Unit and Nursery. The data extracted include the patient's hospital number, culture date, organism isolated and their antimicrobial susceptibilities. We report here the total number of patients with at least one positive blood culture. Rarely two or three sets of blood samples are collected in these patients. When two blood cultures were sent they were counted as 1 patient if they yielded the same organism. If different organisms were cultured they were considered contaminated and not included in the study. The numbers presented here refer to one blood culture per patient. Intrapartum prophylaxis for GBS was not administered to any of the pregnant women during the 11-year review period at both hospitals.

Based on the units' policy, every neonate was investigated according to the sepsis screening protocol (which included complete blood count, differential platelet count and tests for acute phase reactants) and this was usually repeated whenever there was a suspicion of sepsis while in the unit. Screening was either partial (blood and urine cultures) for infants who did not appear ill on admission or complete (blood, urine and cerebrospinal fluid culture) if the infant had definite symptoms of infection such as fever, lethargy or appeared septic at any time. Blood samples (3-5 ml) were obtained after thorough preparation of the skin with iodine solution and cultured by using conventional blood culture procedures [12].

All samples were cultured using an automated culture system (BacT-Alert, bioMérieux, Durham, N.C.; Bectec 9240, Beckton Dickinson, Franklin Lakes, N.J., USA). Bacterial isolates were identified by conventional procedures described in the manual by
Lennette et al. [12]. Antibiotic susceptibility testing was performed using the disk diffusion method described by Bauer et al. [13]. The disks were obtained from Mast Group Ltd., Merseyside, UK. Only antibiotics tested over the entire period were considered for the analysis of multiresistance. Drugs tested for gram-positive bacteria include: penicillin $\mathrm{G}$, fusidic acid, clindamycin, tetracycline, cephalothin, erythromycin, chloramphenicol, vancomycin, and amoxicillin-clavulanate. For gram-negative bacteria ampicillin, ciprofloxacin, chloramphenicol, gentamicin, sulfamethoxazole-trimethoprim, and ceftriaxone were used and for Pseudomonadineae amikacin, ampicillin, aztreonam, ceftazidime, ceftriaxone, chloramphenicol, ciprofloxacin, gentamicin, meropenem, sulfamethoxazole-trimethoprim were used. The standard quality control strains, S. aureus ATCC 25923, Escherichia coli ATCC 25922 and Pseudomonas aeruginosa ATCC 27853, were tested every day, starting in the year 2000. The medium used was Mueller-Hinton agar (Difco; Becton Dickinson, Cockeysville, Md., USA). External quality control was run for the period 1994-2000 with the support of the WHO Collaborative Center for external quality assessment, Belgium. Statistical analysis was done by chi-square test; $p$ value of $<0.05$ was considered as significant.

\section{Results}

\section{Trend of Isolates at SMC 1991-2001}

During the 11-year period, a total of 7,160 newborn infants were admitted to the Neonatal Intensive Care Unit and Nursery in SMC. Of these 288 (4.0\%) had culture-proven bacteremia. The vast majority of them were of Bahraini nationality. No difference was observed between male and female children in susceptibility to infection.

Since 1991 the number of gram-negative bacteria isolated in blood cultures from neonates decreased in a highly significant manner $(\mathrm{p}<0.001)$; while the isolation rate of gram-positive organisms plateaued in the period 1995 2001 at a very high isolation rate (table 1). At SMC isolation rate of gram-positive organisms started to increase sharply in $1995(77.7 \%)$, followed by a minor decline in subsequent years to $69.4 \%$ (table 1). The difference in the positivity between the 1995-1998 and the 1999-2001 intervals was not statistically significant.

Of special concern is the trend in isolation of yeasts, mainly Candida albicans. The organism was present in $2.4 \%$ of patients at SMC 10 years ago to increase threefold in the period 1999-2001, up to 6.5\% (NS) (table 1).

\section{Comparison between the Two Major National \\ Hospitals in the Period 1999-2001}

In BDF Hospital during a 3-year period a total of 818 blood cultures from neonates were processed and 47 (5.7\%) had culture-proven bacteremia, while for SMC 
Table 1. Total positive blood cultures and microorganisms isolated in neonates up to 6 weeks at SMC and BDF

\begin{tabular}{|c|c|c|c|c|c|}
\hline & \multicolumn{3}{|l|}{ SMC } & \multirow{2}{*}{$\begin{array}{l}\text { BDF } \\
1999-2001\end{array}$} & \multirow[t]{2}{*}{ Total } \\
\hline & 1991-1994 & 1995-1998 & 1999-2001 & & \\
\hline Gram-positive blood cultures & 127 & 99 & 62 & 47 & 335 \\
\hline \multicolumn{6}{|l|}{ Gram-positive bacteria } \\
\hline CoNS & 19 & 63 & 35 & 21 & 138 \\
\hline S. aureus & 23 & 4 & 1 & - & 28 \\
\hline GBS & 10 & 7 & 4 & 5 & 26 \\
\hline S. pneumoniae & 3 & 2 & 1 & - & 6 \\
\hline Viridans streptococci & 3 & - & 2 & - & 5 \\
\hline Streptococci non-A-B & - & 1 & - & - & 1 \\
\hline Enterococcus spp. & 6 & 3 & 1 & - & 10 \\
\hline \multirow[t]{2}{*}{ Diphtheroids } & - & 1 & 1 & - & 2 \\
\hline & $64(50.3 \%)$ & $81(81.3 \%)$ & $45(72.5 \%)$ & $26(55.3 \%)$ & $216(64.4 \%)$ \\
\hline \multicolumn{6}{|l|}{ Gram-negative bacteria } \\
\hline E. coli & 25 & 3 & 3 & 4 & 35 \\
\hline Klebsiella spp. & 14 & 6 & 4 & - & 24 \\
\hline Pseudomonas spp. & 1 & 2 & 2 & 1 & 6 \\
\hline Enterobacter spp. & 8 & - & 2 & 7 & 17 \\
\hline Acinetobacter spp. & 4 & 1 & 1 & 1 & 7 \\
\hline Proteus spp. & 3 & - & - & - & 3 \\
\hline Eikenella spp. & - & 1 & - & - & 1 \\
\hline Citrobacter spp. & - & - & 1 & - & 1 \\
\hline Flavobacterium spp. & 4 & - & - & - & 4 \\
\hline Aeromonas spp. & 1 & - & - & - & 1 \\
\hline \multirow[t]{2}{*}{ H. influenzae } & - & 1 & - & - & 1 \\
\hline & $60(47.2 \%)$ & $14(14.1 \%)$ & $13(20.9 \%)$ & $13(27.6 \%)$ & $100(29.8 \%)$ \\
\hline Yeast (C. albicans) & $3(2.4 \%)$ & $4(4.0 \%)$ & $4(6.5 \%)$ & $8(17.0 \%)$ & $19(5.6 \%)$ \\
\hline
\end{tabular}

2,260 blood cultures were examined and $62(2.7 \%)$ showed some growth. The difference is statistically significant $(\mathrm{p}<0.001)$.

In BDF Hospital gram-positive microorganisms were more frequently isolated than gram-negative microorganisms (55.3, 27.6\%, respectively) (table 1), but at SMC isolation rates were 69.4 and $24.1 \%$, respectively (NS). It is evident that in both hospitals, gram-positive organisms were more frequently isolated.

Regarding fungemia, in SMC four cultures (6.5\%) yielded Candida spp. compared to eight (17\%) at BDF (NS). It seems that both institutions have shared characteristics regarding the organisms isolated, indicating a common epidemiology without any statistically significant difference.

\section{Bacterial Isolates at the Two Institutions}

For the sake of clarity the whole data from SMC are included. There were no significant differences between the two major hospitals with regard to the common bac- terial isolates. The main agents isolated (table 1) were coagulase-negative Staphylococcus (CoNS) in 138 cases (41\%) followed by $E$. coli in 35 cases $(10 \%), S$. aureus in 28 cases $(8 \%)$, GBS in 26 cases $(7.8 \%)$, and Klebsiella pneumoniae in 24 cases (7.2\%). Among gram-positive pathogens CoNS were consistently the most common agents isolated, followed by $S$. aureus, and GBS (table 1).

Regarding the role of gram-negative bacteria in neonatal sepsis we decided to concentrate our analysis on the last 3 years in consideration of the sharp decline already mentioned above. E. coli, Klebsiella spp., Enterobacter spp. and Pseudomonas spp. are the most common isolates at both institutions (table 1). Although interesting, yet difficult to explain, variations can be observed, probably the most important single factor being the different antibiotic policies adopted at the two institutions.

For the more recent period, the isolation in decreasing order was CoNS, C. albicans and S. agalactiae; that affected 9 babies during 1999-2001 (4 at SMC and 5 at 
Table 2. Multiresistant strains ${ }^{\mathrm{a}}$ isolated from blood culture at SMC and BDF

\begin{tabular}{|c|c|c|c|c|}
\hline \multirow[t]{2}{*}{ Microorganisms } & \multicolumn{3}{|l|}{ SMC } & \multirow{2}{*}{$\begin{array}{l}\text { BDF } \\
1999-2001^{b}\end{array}$} \\
\hline & $1991-1994^{b}$ & $1995-1998^{b}$ & $1999-2001^{b}$ & \\
\hline \multicolumn{5}{|l|}{ Gram-positive bacteria } \\
\hline CoNS & $8(19)$ & $1(63)$ & $10(35)$ & $19(21)$ \\
\hline Methicillin (R) & 0 & 0 & 0 & 0 \\
\hline S. aureus & $5(23)$ & $0(4)$ & $1(1)$ & 0 \\
\hline Methicillin (R) & 0 & 0 & $1(1)$ & 0 \\
\hline GBS & $2(10)$ & $0(7)$ & $0(4)$ & $0(5)$ \\
\hline Enterococcus spp. & $1(6)$ & $1(3)$ & $0(1)$ & 0 \\
\hline S. pneumoniae & $0(3)$ & $0(2)$ & $0(1)$ & 0 \\
\hline Viridans streptococci & $0(3)$ & 0 & $0(2)$ & 0 \\
\hline Subtotal & $16(64 ; 9.3 \%)$ & $2(79 ; 2.5 \%)$ & $12(45 ; 6.6 \%)$ & $19(26 ; 73.1 \%)$ \\
\hline \multicolumn{5}{|l|}{ Gram-negative bacteria } \\
\hline Citrobacter spp. & 0 & 0 & $1(1)$ & 0 \\
\hline E. coli & $1(25)$ & $0(3)$ & $3(3)$ & $3(4)$ \\
\hline H. influenzae & 0 & $1(1)$ & 0 & 0 \\
\hline Klebsiella spp. & $3(14)$ & $0(6)$ & $0(4)$ & 0 \\
\hline Enterobacter spp. & $1(8)$ & 0 & $1(2)$ & $7(7)$ \\
\hline Acinetobacter spp. & $1(4)$ & $0(1)$ & $1(1)$ & $1(1)$ \\
\hline Pseudomonas spp. & $1(1)$ & $0(2)$ & $0(2)$ & 0 \\
\hline Subtotal & $7(52 ; 13.4 \%)$ & $1(13 ; 7.7 \%)$ & $6(13 ; 46.1 \%)$ & $11(12 ; 91.6 \%)$ \\
\hline Grand total & $23(116 ; 19.8 \%)$ & $3(92 ; 3.2 \%)$ & $18(58 ; 31.3 \%)$ & $30(38 ; 78.9 \%)$ \\
\hline
\end{tabular}

BDF) out of 38,987 deliveries reported (National Health Statistics Annual Reports) (table 1). Time of onset (early vs. late) of the clinical symptoms for GBS was in the late group ( $>1$ week) for the majority of the patients (7 out of 9).

\section{Evolution of Antibiotic Resistance Patterns}

The comparison of the multiresistant strains between the two hospitals is given in table 2. For SMC, data related to the whole study period are reported. Gram-positive resistance showed an apparent increase from $9.3 \%$ in 1991-1994 to $26.6 \%$ in 1999-2001 with identical testing techniques being used over the period (NS). For the most part $S$. epidermidis isolates were sensitive to the antibiotics tested, as was $S$. aureus to methicillin. All GBS strains were sensitive to penicillin $\mathrm{G}$, clindamycin and erythromycin, one was resistant to tetracycline and cephalothin, and one showed resistance to gentamicin.

For gram-negative strains there was a statistically significant increase from $13.4 \%$ in $1991-1994$ to $46.1 \%$ in 1999-2001 $(\mathrm{p}<0.01)$. Klebsiella isolates showed multiple resistances due to the presence of a hospital strain characterized by production of extended spectrum $\beta$-lactamase. High resistance values were detected in isolates from BDF in both gram-positive (73.1\%) and gram-negative organisms $(91 \%)$. The rate of isolation of multiresistant gram-positive as well as gram-negative bacteria was significantly higher in BDF than SMC $(\mathrm{p}<0.001$ and $<0.025$, respectively).

\section{Discussion}

In Bahrain in the recent years CoNS, C. albicans, $S$. agalactiae, Enterobacter spp. and to a lesser extent E. coli constitute the most frequent isolates of neonatal sepsis in the two major hospitals. The overall prevalence of the main pathogens shifted significantly during the study period, the two major findings being a marked decrease in gram-negative isolates contrasted by a sharp increase in isolation rate of yeasts while the isolation of gram-positive pathogens seems to be relatively constant. 
The role of gram-negative pathogens seems conflicting and depending upon each particular institution with some reports of increasing isolation [14] probably linked to specific prophylactic strategies such as widespread use of antepartum antibiotics.

The trend we observed for fungi is similar to the results of other reports [15-18] showing that yeasts are emerging as important nosocomial bloodstream pathogens with possible spread within neonatal intensive care units and thereby posing a potential risk to neonates, including low birth weights. This is the most striking feature of our results, which calls for specific diagnostic and therapeutic policies and guidelines.

Gram-positive organisms are consistently present with CoNS being the most frequent isolates similar to several other studies [4, 6, 7] including Saudi Arabia [6, 7], a neighboring country to Bahrain. As this organism is present on normal skin it is always difficult to ascertain the clinical significance of its occurrence in blood culture, especially if one can, as in our case, rely only on one blood culture bottle. Although the accuracy of the staff in collecting blood was certainly consistent with the recommendations in the manual of clinical microbiology [12] it may not be possible to completely exclude a breach in the technique that could lead to blood contamination. Whereas the general rule of collecting two (or three) sets during $24 \mathrm{~h}$ can be easily applied to adults, it is not always easy to apply it to premature, underweight and severely ill children. Thus, the real clinical relevance of $S$. epidermidis remains in our opinion difficult to assess.

GBS ranked third (when considering the last period) among the isolates with a total of 9 strains $(11.7 \%, 1 / 4,331$ live births) and a total of 26 isolates over an 11-year period $(7.8 \%)$ similar to previous studies $[6,7]$, which is much lower than the reported prevalence in UK or US [14]. We should stress here that intrapartum prophylaxis for GBS neonatal sepsis is not currently applied in Bahrain.

Based on our findings the recommendation of CDC for prevention of GBS disease including universal prenatal screening for pregnant women seems not to be appropriate for our epidemiological setting. The burden and the cost of effecting the policy will require a careful discussion before being implemented if one estimates 30,000 specimens/year - including vaginal and rectal swabs for 15,000 pregnant women - this will add up to 75,000 Euro to prevent 3 infections in a year. Therefore we recommend prenatal screening in the presence of additional risk factors for GBS disease $[19,20]$ such as: longer duration of membrane rupture, maternal age, presence of GBS at previous testing or previous delivery of a baby with GBS disease. Indeed some of the above can be considered indications for starting intrapartum prophylaxis without waiting for the microbiological report.

A related issue is the need to test the GBS strains for antibiotic sensitivity. According to our sensitivity profile, penicillin $\mathrm{G}$ seems to maintain its full effectiveness and no clindamycin or erythromycin resistance has been detected. Again we feel that CDC guidelines for GBS testing are not applicable in our situation as a routine approach although it might be of interest to run Gulf collaborative studies to monitor the evolution of resistance in these important pathogens.

Of greater concern is that CoNS showing resistance to more than three antibiotics rose from 1.5\% in 1995-1998 to $28 \%$ in $1999-2001$ in SMC and to $90 \%$ in BDF. The difference between the two hospitals for the same time interval was statistically highly significant ( $\mathrm{p} \leq 0.001)$. Enterobacter and Acinetobacter species also showed worrying high rates of multiple resistances. This trend among gram negatives is disquieting and requires careful observation, stressing the constant need for rigorous policies and incessant education for proper antibiotic use in hospitals. A supplementary major finding of this paper relevant for establishing national policies is the significantly higher rate of isolation of multipliresistant strains at BDF. Although this can partially be explained with the dissimilar patient population, nevertheless there is an urgent need for unified national guidelines in support of appropriate antibiotic use.

\section{Conclusion}

Good quality specimens are essential to evaluate the role of CoNS. The increasing threat of fungal infection must be carefully tackled. Specifically tailored policies for GBS prevention must be defined according to local epidemiology.

\section{Acknowledgments}

This work has been partially supported by a research grant to Prof. Giuseppe A. Botta from the Arabian Gulf University. We are indebted to Dr. Abiola C. Senok for critically reviewing the manuscript and to Tomy Kaitharath for secretarial help. 


\section{References}

1 Polin RA, Geme JW III: Neonatal sepsis. Adv Pediatr Infect Dis 1992; 7:25-61.

$\checkmark 2$ Greenough A: Neonatal infection. Curr Opin Pediatr 1996;8:6-10.

-3 Kaufman D, Fairchild KD: Clinical microbiology of bacterial and fungal sepsis in very-lowbirth-weight infants. Clin Microbiol Rev 2004; 17:638-680.

$\checkmark 4$ Centers for Disease Control: Prevention of perinatal group B streptococcal disease: a public health perspective. MMWR Morbid Mortal Wkly Rep 1996;45(RR-7):1-24.

$\checkmark 5$ Walsh JA, Hutchins S: Group B streptococcal disease: its importance in the developing world and prospect for prevention with vaccines. Pediatr Infect Dis J 1989;8:271-276.

-6 Dawodu A, al Umran K, Twum-Danso K: A case control study of neonatal sepsis: experience from Saudi Arabia. J Trop Pediatr 1997; 53:84-88.

7 Kilani RA, Basamad M: Pattern of proven bacterial sepsis in a neonatal intensive care unit in Riyadh-Saudi Arabia: a 2-year analysis. J Med Liban 2000;48:77-83.

$>8$ Karthikeyan G, Permkumar K: Neonatal sepsis: Staphylococcus aureus as predominant pathogen. Indian J Pediatr 2001;68:715-717. $\checkmark 9$ Cordero L, Sananes M, Ayers LW: Bloodstream infections a neonatal intensive-care unit: 12 years' experience with an antibiotic control program. Infect Control Hosp Epidemiol 1999; 20; 242-246.

10 Joshi SG, Ghole VS, Niphadkar KB: Neonatal gram-negative bacteremia. Indian $\mathrm{J}$ Pediatr 2000;67:27-32.

11 Bromiker R, Arad I, Peleg O, Engelhard D: Neonatal bacteremia: patterns of antibiotic resistance. Infect Control Hosp Epidemiol 2001; 22:767-770.

12 Lennette EH, Balows A, Hausler WJ Jr, Shadomy JH (eds): Manual of Clinical Microbiology, ed 4. Washington, American Society for Microbiology, 1985.

13 Bauer AM, Kirby WMM, Sherris JC, Turck M: Antibiotic susceptibility testing by a standardized single disc method. Am J Clin Pathol 1966;465:493-496.

14 Towers CV, Carr MH, Padilla G, Asrat T: Potential consequences of widespread antepartal use of ampicillin. Am J Obstet Gynecol 1998; 179:879-883.
15 Beck Sague C, Jarvis WR: Secular trends in the epidemiology of nosocomial fungal infection in the USA 1980-1990. J Infect Dis 1993;167: 1247-1251.

16 Reagan DR, Pfaller MA, Hollis RJ, Wenzel RP: Evidence of nosocomial spread of Candida albicans causing bloodstream infection in a neonatal intensive care unit. Diagn Microbiol Infect Dis 1995;21:191-194.

17 Stamos JK, Rowley AH: Candidemia in a pediatric population. Clin Infect Dis 1995;20: 571-575.

18 Reef SE, Lasker BA, Butcher DS, McNeil MM, Pruitt R, Keyserling H, Jarvis WR: Non-perinatal nosocomial transmission of Candida albicans in a neonatal intensive care unit: prospective study. J Clin Microbiol 1998;36: 1255-1259.

19 Wessels MR, Kasper DL: Group B streptococci; in Gorbach S, Bartlett JG, Blackleg NR (eds): Infectious Diseases. Philadelphia, Saunders, 1992, pp 1421-1424.

20 Oddis S, Embleton ND: Risk factor for early onset neonatal group B streptococcal sepsis: case control study. BMJ 2002;325:308-311. 